\title{
URBAN HEAT ISLAND FOOTPRINT EFFECTS ON BIO-PRODUCTIVE RURAL LAND COVERS SURROUNDING A LOW DENSITY URBAN CENTER
}

\author{
Michael Burnett* and Dongmei Chen
}

Department of Geography and Planning, Queens University, Kingston, Canada

KEY WORDS: Urban Heat Island Footprint, Landsat 7 Enhanced Thematic Mapper, Surface Urban Heat Island, Land Surface Temperature, Low-Density, Normalized Difference Moisture Index, Vegetation Index.

\begin{abstract}
:
The urban heat island (UHI) is a common effect caused by urbanization and has been studied to evaluate the thermal condition in cities worldwide. However, most previous UHI analyses are performed in major metropolitan cities. This study conducts a spatiotemporal analysis of UHI in a rapidly expanding low-density suburban centre and determines how bio-productive land covers react and the extent of the disturbance to each land cover based on time series land surface temperatures extracted from Landsat 7 ETM+ images. Two methods applied and compared are the single exponential decay method, which measures UHI footprint (UHIFP) on vegetation phenology, and the two dimensional Gaussian surface, which quantifies the influence based on distance from the local urban perimeter. Three spectral indices (Normalized Difference Vegetation Index (NDVI), Moisture Index (NDMI), and the Enhanced Vegetation Index (EVI)) were extracted and the residuals from the Gaussian model were compared based on these indices in order to better understand the thermal variations of each land cover within a UHI. The results show that the UHIFP of the studied low-density suburban centre is 1.4 times larger than the size of the urban centre, marginally smaller than previous analyses performed within high-density metropolises. All vegetated land covers experienced their maximum cooling effects before reaching the UHIFP perimeter while urban surfaces begin to diverge from the Gaussian model outside of the UHIFP. The residuals of sparse vegetation maintained strong correlations with each index throughout the growing season while NDMI retained the strongest relationships with every land cover. This study has helped us better understand the UHI effects of small communities with varied vegetation phonology based on the distribution of built-up pervious and impervious surfaces within the neighbourhood structure. The similar results from both methods indicate a strong urban cover influence overpowering the dominant distribution of agricultural surfaces throughout the growing season.
\end{abstract}

\section{INTRODUCTION}

The urban heat island (UHI) is the product of anthropogenic processes with urbanization which modifies atmospheric and surface properties and alters the energy balance and thermal environment (Yang, Huang, and Tang 2019). It has been studied as far back as early 19th Century (Howard 1818) and is a wellexplored phenomenon with urban centres of varying sizes (Oke 1973; Katsoulis and Theoharatos 1985; David R Streutker 2003; Krehbiel, Jackson, and Henebry 2016; Yao et al. 2019).

Thermal infrared (TIR) data quantified from top of atmosphere radiances in satellite imagery is used to derive land surface temperatures (LST) (Tomlinson et al. 2011). UHI research, now commonly measured globally using LST, has accelerated since 2005 with the majority of regional and seasonal focus being in China and summer daylight hours (D. Zhou et al. 2019). Using the UHI intensity measured between urban temperatures and a referenced rural region, the footprint of the UHI effect is a new index quantifying the spatial extent of the rural area affected by the UHI (Qiao et al. 2019).

Two commonly used approaches for determining the spatial influence of the UHI are the urban heat island footprint (UHIFP) and the footprint of surface urban heat island (SUHI) model developed by D. R. Streutker (2002; 2003). The UHIFP, first introduced to measure the anthropogenic effects that urbanization has upon the warming of vegetation phenology (X. Zhang et al. 2004), has been adapted to determine the distance at which the UHI effects decay towards rural areas (D. Zhou et al. 2015). The footprint model of SUHI effect uses a Gaussian planar surface to fit the UHI effect and has been widely applied to study the UHI in many metropolitan areas (D. R. Streutker 2002; David R Streutker 2003; Martin, Baudouin, and Gachon 2015; Sobrino et al. 2012; Anniballe, Bonafoni, and Pichierri
2014; Anniballe and Bonafoni 2015; Yao et al. 2017; Qiao et al. 2019; D. Zhou et al. 2019). However, the footprints of both approaches have not been compared.

The majority of previous UHI studies using remote sensing are performed with mean annual data from daily products using coarse spatial resolution sensors such as the Moderate Resolution Imaging Spectroradiometer (MODIS) (Yao et al. 2017; Qiao et al. 2019). However, the smoothing effects with high-resolution thermal data are more appropriate for smaller scales despite having higher UHI magnitudes and spatial extents (Anniballe and Bonafoni 2015). Furthermore, the land cover products used in this analysis were also derived from Landsat which will help produce robust residual analyses based on each bio-productive surface.

The application of UHI studies in a cold and urban country such as Canada is increasingly necessary. Climate models indicate greater warming in colder regions, accelerated transformations of nitrogen oxides and volatile organic compounds into ozone, and the population's sensitivity to heat higher than $20^{\circ} \mathrm{C}$ (Y. Wang, Berardi, and Akbari 2016). With most of the research focused on major metropolitan cities (Touchaei and Wang 2015; Y. Wang, Berardi, and Akbari 2016; Adamowski and Prokoph 2013), the UHI effect of low-density suburban centers, where the majority of Canadian urban growth occurs (Maoh and Kanaroglou 2007), are often not assessed individually.

This study examines the UHI effect of a low-density suburban centre near the Greater Toronto Area (GTA) from 2000 to 2019 using time series images of Landsat 7 Enhanced Thematic Mapper (ETM+). By comparing the footprint produced from both models, this analysis seeks to address three main questions: 1) what is the extent of thermal disturbance of the

\footnotetext{
* Corresponding author
} 
UHI effect to each bio-productive land cover in the surrounding rural areas?, 2) are the calculated footprints from UHIFP and SUHI consistent?, and 3) what are primary factors influencing the spatiotemporal pattern that a low-density suburban centre has upon differing land covers with varying distance from the urban perimeter?

\section{MATERIALS}

\subsection{Study Site}

The location of this study is in Milton, Ontario, Canada. It is situated within the GTA, the most populous area throughout Canada, and is surrounded by a plethora of agricultural and urban land covers, as well as some forested and wetlands. Milton's population has risen $30.5 \%$ to 110,128 between 2011 and 2016 making it the most rapidly growing community in Eastern Canada (Hennessey 2017).

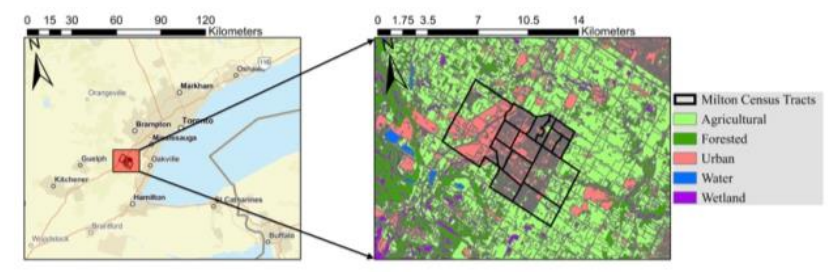

Figure 1. The distribution of land covers surrounding Urban Milton, its designated census tracts, and its location within the GTA in the 2015 land cover product.

Urban Milton (UM) and Rural Milton are defined based on population density due to their relatively homogenous neighbourhood structures and their stability for time-series analyses (Gordon and Janzen 2013). Figure 1 displays in boldened polygons the appropriate census tracts representing UM for which the urban surfaces within are representative of the urban centre. Gordon and Janzen (2013) defined urban census tracts as having a population density in excess of 150 people/km2 and exurban having a low gross population density and heavy reliance upon automobiles where over half of the labour force commutes to central cities for employment. This explains 13 of the census tracts; however, a fourteenth tract was selected due to its wide distribution of industrial urban surface components.

\subsection{Land Cover Data}

Version 3.0 of the Southern Ontario Land Resource Information System (SOLRIS) includes land cover maps in 2002, 2006, 2011, and 2016 in the Milton area with a pixel resolution as low as $15 \mathrm{~m}$ (Science and Research Branch of the Ministry of Natural Resources and Forestry 2019). Landsat imagery is associated with the nearest chronological land cover product (2004 imagery is analysed with the 2006 land cover product). The SOLRIS dataset employs methodology presented by Lee et al. (1998) to optimize the implementation of the classification techniques. It presents reliable land cover products with overall accuracy of $93 \%$ based on an assessment conducted within a polygon encompassing the study area (Sampson 2007).

19 unique classes existing within close proximity to Milton were grouped into five land cover categories, including open water. Table 1 displays the four merged classes; wetlands (any surface where the water table is either seasonally or permanently at, near, or above the substrate surface), forest, agriculture (including undifferentiated land covers in reference to alternative tilled surfaces not included in the 'tilled' class, hydro fields, forest openings, and brownfields), and urban (Science and Research Branch of the Ministry of Natural Resources and Forestry 2019).

\begin{tabular}{|c|c|c|c|}
\hline Agricultural & Urban & Forested & Wetlands \\
\hline $\begin{array}{c}\text { Plantations - } \\
\text { Tree Cultivated }\end{array}$ & Transportation & Forest & $\begin{array}{c}\text { Treed } \\
\text { Swamp }\end{array}$ \\
\hline Hedge Rows & $\begin{array}{c}\text { Built-Up Area } \\
\text { - Pervious }\end{array}$ & $\begin{array}{c}\text { Coniferous } \\
\text { Forest }\end{array}$ & $\begin{array}{c}\text { Thicket } \\
\text { Swamp }\end{array}$ \\
\hline Tilled & $\begin{array}{c}\text { Built-Up Area } \\
\text { - Impervious }\end{array}$ & $\begin{array}{c}\text { Mixed } \\
\text { Forest }\end{array}$ & Marsh \\
\hline $\begin{array}{c}\text { Undifferentiated } \\
\text { (orchards, }\end{array}$ & & & \\
\hline
\end{tabular}

Table 1. The reclassification of all land covers into four manageable categories. A fifth classification for water is not included as it was not reclassified.

\subsection{Remote Sensing Data Acquisition}

In order to maintain data consistency, only LST data acquired solely from Landsat 7 ETM+ from 2000 to 2019 were used in this study. The ETM+ imagery has 7 main bands (along with an additional panchromatic band) with spatial resolutions ranging from 15 to 60 meters, and a 16-day temporal resolution (National Aeronautics and Space Administration 2020). The LST is extracted using the statistical mono-window algorithm (SMW) conceived by Ermida et al. (2020) from atmospherically corrected surface reflectance Landsat $7 \mathrm{ETM}+$ products in Google Earth Engine (Malakar et al. 2018). Its accuracy meets the threshold proposed by Guillevic et al. (2018) with overall precision (in RMSE) of $1.0 \mathrm{~K}$ determined by Ermida et al. (2020). A $20 \%$ local cloud coverage threshold was used to select appropriate LST images for UHI analysis.

2.3.1 Seasonality: The annual timeframe used in this study was based around the growing season due to the complexity of environmental variables with the local climate. Soil moisture, snow cover, cloud cover, atmospheric moisture, precipitation, and anthropogenic gases are among the elements widely studied to separate the typical extremes of summer and winter measurements (Bernhardt and Carleton 2019; K. Wang et al. 2017; W. Zhou et al. 2014). Late autumn and winter data present far too many obstructions and interference caused by snow coverage. As a result, the annual calendar days included in the research consists of days 100 (April 9/10) to 290 (October 16/17). These days exhibit minimal frost and snow coverage along with increasing solar radiation and vegetated growth (Ministry of Agriculture, Food and Rural Affairs 2020). In order to differentiate the portions of the growing season for this analysis, April and May (referred to as 'Spring') are combined representing the end of frost coverage and the initial planting phase, June through August is the main summer period, and September and October (referred to as 'Autumn') represent the harvest and end of season.

\section{METHODS}

\subsection{UHIFP}

UHIFP uses a single exponential decay model as the following to examine temperature trends from an urban center toward rural areas (D. Zhou et al. 2015).

$$
\Delta \mathbf{T}=\mathbf{A} * \mathbf{e}^{-\mathbf{s}^{*} \mathbf{D}}+\mathbf{T}_{0}
$$


where $\quad \Delta T=$ mean LST differences between the urban area and rural buffers

$\mathrm{A}=$ maximum temperature difference using the midvalue amongst the three furthest rural buffers

$\mathrm{S}=$ temperature decay rate

$\mathrm{D}=$ distance away from the urban centre

$\mathrm{T}_{0}=$ asymptotic value

To determine the spatial properties of the UHI impact on rural surfaces, buffers are implemented as a factor of distance from the urban perimeter. In order to maintain a similar quantity of pixels within each buffer zone, twelve buffers surrounding the urban land cover area within UM census tracts are generated, each consisting of half the urban surface area (Zhou et al., 2015). Transportation surfaces are masked out to avoid the inclusion of roads in the rural parts of UM census tracts. Instead, a $15 \mathrm{~m}$ buffer around all other urban surfaces is used to regulate a centralized urban centre. As this study uses four land cover products addressing Milton's growth over 20 years, a different set of buffers are generated consistently maintaining a total rural area 6 times larger than the urban centre.

Previous research examined annual mean UHIFP based on daily MODIS products (X. Zhang et al. 2004; D. Zhou et al. 2015; C. Meng and Dou 2016). Due to the exchange of a 16 days revisit cycle of ETM+, a 5-years period is implemented with the central year determining the land cover product. With all 16 time periods (period $1=2000-2004$ ), each set of results are inputted into the single exponential decay model.

All cloud cover pixels and elevations greater than $50 \mathrm{~m}$ from the highest point in UM were removed from the UHIFP model. The footprint determination is based upon the point at which each exponential model reaches $95 \%$ of their asymptotic values (X. Zhang et al. 2004). The final product represents the area surrounding UM affected by the local UHI within the proximate exurban buffers.

\subsection{SUHI Gaussian Fit Model}

The planar fit Gaussian surface was developed through the method described by D. R. Streutker $(2002 ; 2003)$ to quantify the UHI as a continuously varying surface. Its purpose is to present a quick and robust technique for analysing and parameterizing the spatial distribution of the SUHI for quantitative inter-city comparisons or single city time scale analyses (Anniballe and Bonafoni 2015). To access the Gaussian surface, the following equation is used:

$$
\begin{array}{r}
\operatorname{SUHI}(x, y)=a_{0} * \exp \left[-\frac{\left(\left(x-x_{0}\right) \cos \varphi+\left(y-y_{0}\right) \sin \varphi\right)}{0.5 a_{x}^{2}}\right. \\
\left.-\frac{\left(\left(y-y_{0}\right) \cos \varphi+\left(x-x_{0}\right) \sin \varphi\right)}{0.5 a_{y}^{2}}\right]
\end{array}
$$

where $\quad x_{0}, y_{0}=$ central location of the model's UHI

$$
\begin{aligned}
& a_{0}=\text { magnitude } \\
& a_{x}, a_{y}=\text { spatial extent } \\
& \varphi=\text { orientation }
\end{aligned}
$$

All cloud and water pixels must first be masked out from the LST image followed by the temporary removal of urban pixels within UM to produce datasets consisting entirely of rural pixels. After acquiring the mean rural temperature, it is then subtracted from the entire LST image to generate the SUHI Gaussian surface.
Its results produce two elliptical thresholds representing the footprint of the SUHI in each image. One ellipse determines the distance from the central location at which the temperature decreases to a fraction of the magnitude, $61 \%\left(\mathrm{e}^{-1 / 2}\right)$ of the maximum value, and the second ellipse is a constant threshold where the SUHI planar fit surface temperature is greater than 1.0 K (Anniballe and Bonafoni 2015).

3.2.1 Residuals Analyses: The SUHI planar fit model does not produce thresholds that align with the shapes of either UM or the rural buffers produced for the UHIFP model. Alternatively, the Gaussian surface produces residuals which can be analysed with the buffers and a land cover distribution map. Four analyses are made regarding the residuals' relationship with the planar surface; a ratio analysis, a buffer comparison, and a timeframe analysis.

The ratio analysis divided the quantity of positive residuals by the negative and the results with each land cover are displayed in a box-plot separated by months. Its purpose is to quantify the relationship that pixels within each land cover have with the planar fit model depending on whether the residuals are above or below the SUHI surface.

The Gaussian surface's residuals are also analysed using the buffer areas applied to the UHIFP model. The buffer analysis helps determine the SUHI model's spatiotemporal influence and provides a spatial analysis comparable with the UHIFP. Seasonal divisions are implemented based on vegetation growth and solar radiation separating the beginning, middle, and end of the growing season. The mean seasonal distribution of residuals for land covers within each buffer is compared.

The time frames used for each distribution of land covers are incorporated into a time frames analysis. Using Google Earth Engine, mean imagery for each year associated with a SOLRIS product was calculated and compared. General UHI magnitudes, spatial extents, and Gaussian surface residuals are analysed for each part of the growing season. Because of the nature of this analysis, a series of complications occur such as the inability to control local cloud coverage and a bias towards the few days with clear skies. These results are taken with caution and only conclusions regarding general UHI trends are made.

\subsubsection{Vegetation Health and Moisture Correlation} Analyses with Residuals: Understanding the variables affecting the residuals of each bio-productive land cover in the SUHI model is achieved through correlation analyses between the residuals and vegetation and moisture indices. The multispectral Landsat 7 bands required from each date are blue, red, near infrared (NIR), and shortwave infrared (SWIR). Amongst the vegetation indices, NDVI is more commonly used due to its cancellation of topographic, cloud cover, atmospheric, and changing sun angle effects with vegetation monitoring (Alademomi et al. 2020).

$$
\text { NDVI }=\frac{(\text { NIR }- \text { Red })}{(\text { NIR }+ \text { Red })}
$$

Enhanced Vegetation Index (EVI) acts as an enhancement to it with its atmospheric correction, saturation in areas with high biomass, and soil reflectance reduction abilities (Alademomi et al. 2020).

$$
E V I=2.5 * \frac{(\text { NIR }- \text { Red })}{\left(\text { NIR }+6^{\star} \text { Red }-7.5 * \text { Blue }+1\right)}
$$


Normalized Difference Moisture Index (NDMI) behaves as an index to reflect the moisture content within the soil and vegetation canopy (Zhu et al. 2019).

$$
\text { NDMI }=\frac{(\text { NIR }- \text { SWIR })}{(\text { NIR }+ \text { SWIR })}
$$

The comparisons made with vegetation indices use Landsat 7 ETM+ Surface Reflectance Tier 1 products on the same dates as the LST acquisition. A cloud mask was installed, the residuals' pixels were converted to points, and all values from each vegetation/moisture index were extracted to the nearest point. The correlation coefficient is calculated with a range of 1.0 (positive correlation) to -1.0 (negative correlation).

\section{RESULTS AND DISCUSSION}

\subsection{UHIFP}

The results from the UHIFP are determined based on the distance at which the exponential model reaches $95 \%$ of its asymptotic value. For Milton during the growing season, the mean UHIFP is achieved at 1.4 times the urban area (Figure 2). As a result, the first three buffers are representative of the area within the local average UHIFP. The strongest outliers were the first two time frames (2000-2004 and 2001-2005) with marginally increased footprints (1.8 times the size of the urban centre).

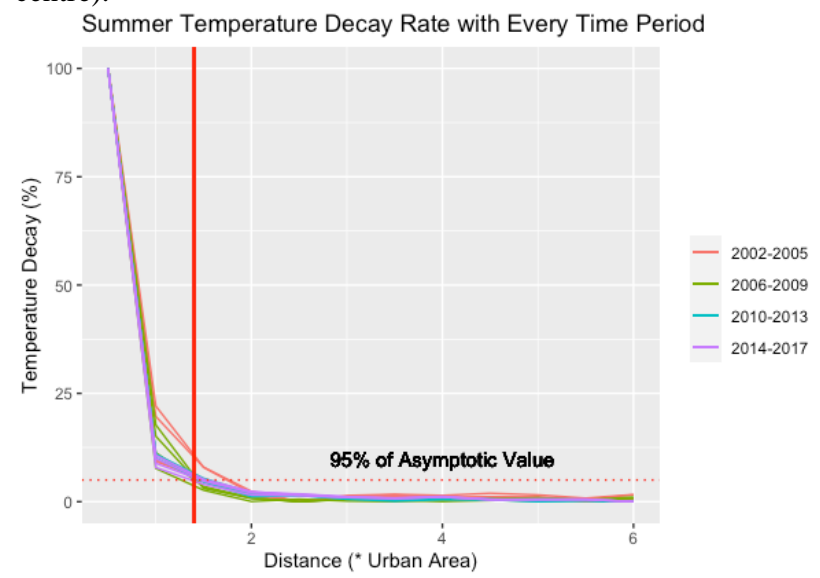

Figure 2. The determined size of the UHIFP, where the temperature declines to $95 \%$ of the asymptotic value, is 1.4 times the urban area of Milton based on an overall average.

The size of the UHIFP within each set of mean sampled imagery was consistent after the first two time frames. The results experienced in Milton are much smaller than the conclusions drawn by X. Zhang et al. (2004), which were 2.4 times the area of an urban centre in eastern

North America. Such results are related to the local conditions influencing the UHIFP analysis, such as population density, city size, proximity to a larger urban centre, and the surrounding vegetation distribution.

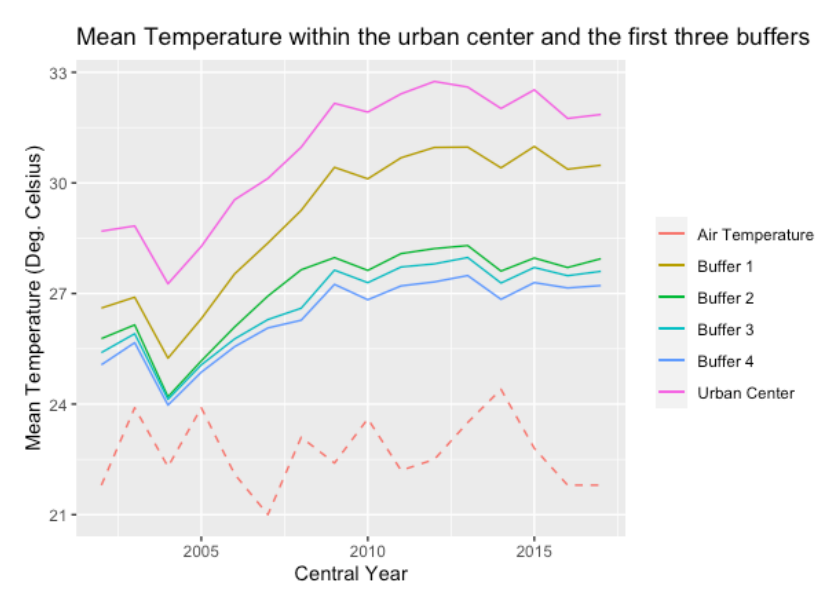

Figure 3. The mean LST collected within each time period within the urban centre and the first four buffers. Daily air temperature data from the nearest station to Milton, in Georgetown, is applied to separate the local UHI effects from regional climate change.

Figure 3 presents the mean LST within the urban centre and the adjacent rural area 2 times the urban centre's size (first four buffers). The temperature difference between the urban centre and the first buffer exhibits minimal change while an increasing difference is evident between the first and second buffers after 2005. Following the second buffer, the mean temperature differences amongst buffer regions remain consistently small while most UHI effects have decayed. The data in Figure 3 presents mean LST increasing at a higher rate within the urban centre and the first buffer than the following buffers. As a result, the UHIFP of the very small low-density community, prior to its expansion, is larger relative to the urban centre's area.

The proportion of each land cover within the local UHIFP compared to its distribution throughout the area 6 times the size of UM can help determine the urban centre's impact on each type of surface. The portion of forested surfaces within the footprint was between $11 \%$ and $14 \%$ of its total. Wetlands and agricultural land covers contain slightly higher proportions within the UHIFP (19\% - 22\% and $24 \%-27 \%$ respectfully) The majority of urban surfaces were contained within the footprint (between $65 \%$ and $73 \%$ ); however, exclusive of the urban centre, only between $18 \%$ and $27 \%$ of the rural urban surfaces were located inside the footprint. 
4.1.1 UHIFP Size Discussion: The population arrangement and overall size of an urban centre are foundational in determining the footprint of a UHI. Population density can have a major effect on temperature differences within a city as it grows (Ramírez-Aguilar and Lucas Souza 2019). An increased distribution of impervious surfaces, coinciding with rising human activity frequency, contribute to the growing intensity of a UHI (F. Meng and Liu 2013). In comparison with previous studies applied to larger municipalities with denser population distributions, Milton's suburban stature is incapable of reproducing the increasing trends of sensible heat flux experienced in larger cities with less vegetated surfaces.

The proximity to much larger urban centres heavily affects the determination of the local UHIFP. All of the surrounding UHI footprints influence the referenced rural temperatures used for Milton's analysis. For example, the first two time periods (2000-2004 and 2001-2005) determined larger footprints due to Milton's smaller urban size and a reduced external influence from neighbouring UHI footprints. Milton's growth coincides with neighbouring communities' development and their influence on rural temperatures. As a result, an inevitable discrepancy is found between urban and rural temperatures in a suburban setting compared with a larger metropolis, being the primary influence on rural temperatures.

4.1.2 Vegetation Distribution within the UHIFP: The vegetation distribution surrounding Milton is another major variable on its ecological impact upon the surrounding temperatures. While urban areas surrounding forested or highly vegetated areas produce a greater discrepancy in UHI, partly due to residential energy consumption for cooling during the summer (Imhoff et al. 2010), Milton's rural land covers consist largely of sparse vegetation. The Niagara Escarpment, a large nearby forested area, was omitted from the rural UHIFP due to the impact of elevation. Additionally, being an inland town with limited open water and wetlands surfaces in close proximity may result in lower evapotranspiration comparisons between urban and rural (D. Zhou et al. 2015; Li, Zhang, and Kainz 2012). As a result, urban centres lacking nearby dense vegetation may experience reduced UHIFP sizes.

The diminutive proportion of the study area's dense vegetation located within the UHIFP, as opposed to its distribution in the outer buffers, can have a major influence on the UHI effect on rural LST. Its cooling effects and spatial distribution have the ability to reduce the footprint encroachment on rural vegetation. Additionally, as the UHIFP contains a quarter of the local agricultural surfaces and the majority of urban surfaces, the spatial variability of vegetated land covers, and concentration of impervious surfaces, is a major contributor to a smaller footprint size.
4.1.3 UHIFP Limitations: The limitations within this study for calculating the UHIFP with Landsat 7 ETM+ consists of an optimistic 16 days revisit cycle, only one time of day where imagery acquisition is possible, and the distribution of snow and ice surfaces in the winter. Previous research (D. Zhou et al. 2015) utilized MODIS products due to their near daily data availability, possibility for comparing daytime and night-time data, and much larger metropolitan sizes. In comparison, Milton's urban area is dwarfed by the far larger North American and Chinese cities. The resulting 5 years periods applied to determine mean pixel values for a UHIFP is unable to reproduce daily samples possible with MODIS analyses; however, this study's results were consistent with every period. Until a satellite is launched with similar spatial resolutions as Landsat and a major reduction in its revisit cycle, applying a 5 years average may presently be the optimal solution as uniform results were found within each 5 years period. With winter conditions, the model remains sufficient provided the urban area is a large enough size.

\subsection{SUHI Model}

The SUHI model was generated from a limited quantity of image acquisition dates due to a coarse temporal resolution and cloud interference. From the available results, the SUHI magnitude observes a rising trend $(0.16 \mathrm{~K} /$ year $)$. From Figure 4 , it can be seen that the area with $1.0 \mathrm{~K}$ UHI threshold is increasing at a much more dramatic rate of 3626.7 pixels/year compared with the $\mathrm{e}^{-1 / 2}$ UHI threshold (a slope of 559.5 pixels/year).

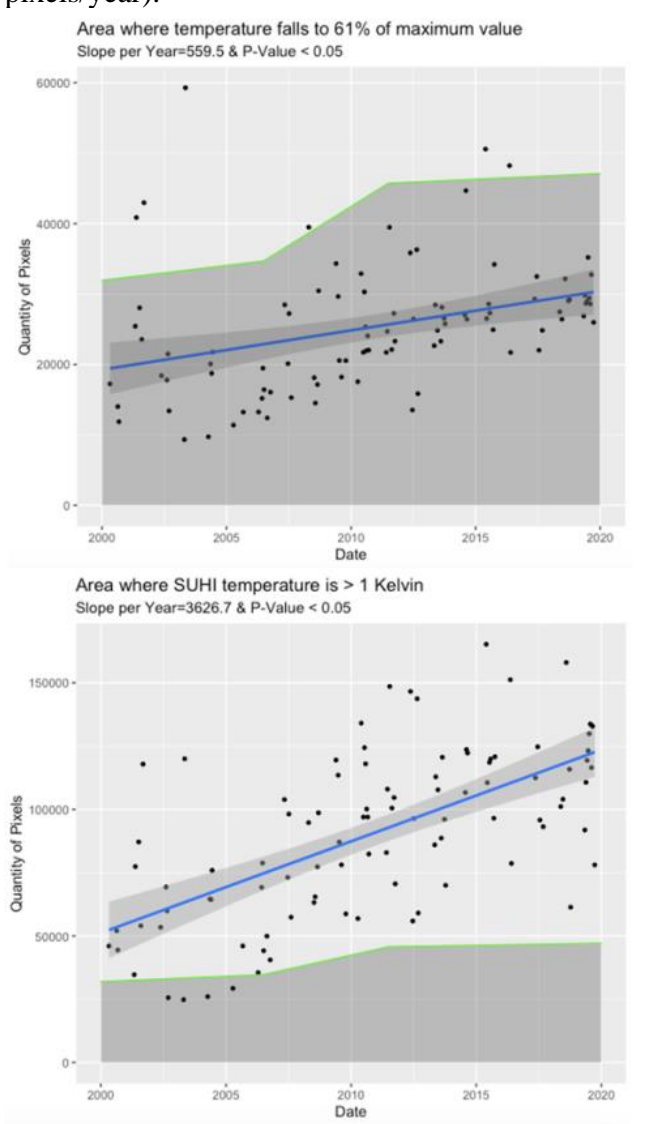

Figure 4. The size of both SUHI thresholds from each acquired image. The total quantity of urban pixels within Urban Milton (green) and the line of regression (blue) are presented to compare planar surface SUHI threshold results with actual urban area. 
The surface thresholds indicated rising trends, and in the case of the $1.0 \mathrm{~K}$ temperature ellipse (Figure 4), greatly surpassing the area of UM. The rate at which the thresholds grow appears to align with the rate of expansion of the built-up environment. Based on the distribution and quantity of urban pixels within UM for each land cover product, the timeframe between 2006 and 2011 is when UM experienced its greatest degree of urban development. The declining vegetated surfaces along with increasing impervious surface, anthropogenic gases, and population densities are all highly correlated with higher surface temperature, especially during the urbanization process ( $\mathrm{Lu}$ et al. 2015; Yu et al. 2019). The development of new built-up environments result in increased volume of ozone, faster pollutant production lofted to higher altitudes, and greater transport of the adverse effects on air quality (D. L. Zhang et al. 2011). Further evidence of these effects are displayed in the Table 2 time frames comparison where the most dramatic changes in magnitude occurs between 2004-2008 and 20092013 (with the exception of autumn where observations were in minimal supply).

Time series results incurred from the overall SUHI data, along with the time frames analysis with residuals data, must be taken with speculation due to the insufficient temporal resolution of the Landsat 7 ETM+ sensors. In order to accurately determine the SUHI effects, a spatial resolution similar to Landsat is required along with a 2 days revisit cycle and the overpass time immediately before sunrise (Sobrino et al. 2012). There are currently no spaceborne thermal sensors which satisfy these requirements. The available products from Landsat 7 meet the spatial resolution requirements; however they are heavily unqualified for the temporal obligation. Although trends are noticeable, conclusions based on magnitudes are incapable of being drawn due to insufficiencies with the products.

4.2.1 Residuals Ratio Analysis: Figures 5 and 6 list the ratio between quantities of positive and negative residuals in each month for each land cover type. The results from the ratio analysis indicate the distinctive impact of urban cool islands within the forested and wetlands surfaces surrounding UM. April and May data describe residual cooling behaviour less intense than in the summer and early autumn.

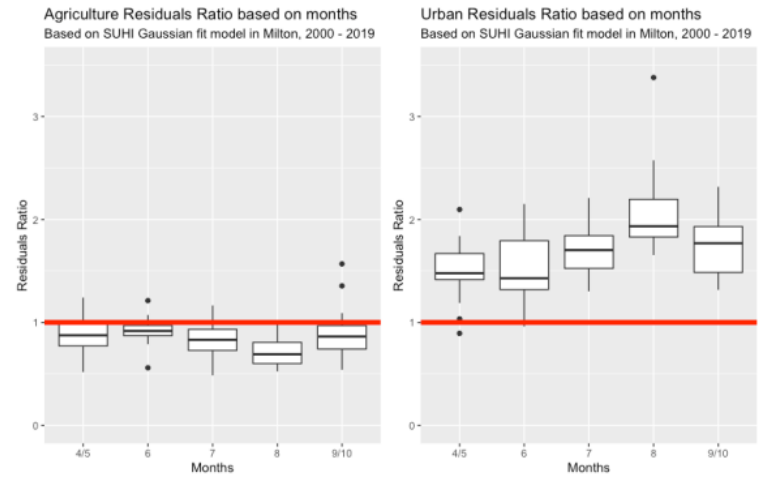

Figure 5. The residuals ratio for agriculture and urban land covers determined by dividing the number of positive residuals by negative residuals within each captured Landsat 7 ETM+ product.
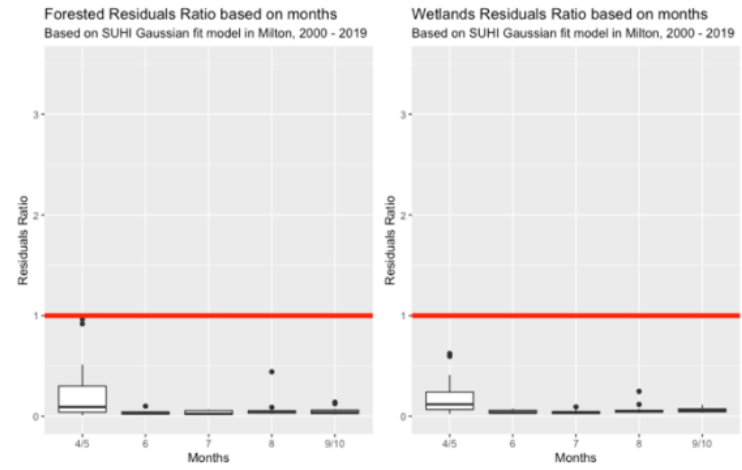

Figure 6. The residuals ratio for forested and wetlands land covers determined by dividing the number of positive residuals by negative residuals within each captured Landsat 7 ETM+ product.

The most interesting results in this analysis are within the agricultural and urban surfaces. The agricultural residuals, being the dominant land cover throughout the study site, follows the Gaussian planar surface closely with only a marginally greater quantity of negative residuals. Spring and Autumn months exhibit results which better align with the SUHI model as opposed to summer months where a much more definitive distribution of negative residuals exist. The cooling effects climax in August with the average ratio being 0.72, as seen in Figure 5. The urban results contrast vegetated surfaces where positive residuals consistently outnumber negative residuals. As summer progresses, the distribution of positive residuals grows to an August peak.

It is well established that vegetated land covers behave as cooling zones within a UHI (Oke 1979; Sun, Wu, and Tan 2012; Li, Zhang, and Kainz 2012; Skelhorn, Levermore, and Lindley 2016; Q. Huang et al. 2019; Alademomi et al. 2020). The ratio analysis results in Figure 5 further exemplify that notion with only the urban land cover category obtaining a greater ratio of positive residuals compared to negative residuals. As summer progresses and thermal emissivity and capacity on impervious surfaces increases, the divergence urban land covers' residuals have compared to the vegetated covers increases (H. Huang et al. 2019). The cooling effects from vegetated land due to evapotranspiration are largely removed when replaced with impervious materials (Oke 1979). The result is a surface with a heavily limited ability for evapotranspiration, and cooling properties, culminating in a warmer surface temperature compared to vegetation.

The crop surfaces in spring months (April and May) along with the autumn months of September and October (to a lesser degree) exhibit a reduction in its cooling abilities within the UHI. With the possibility of frost as late as May and cultivation occurring in September, the agricultural results in Figure 5 display considerable distributions of bare earth at the beginning and end of the growing season. The summer months of July and August, when sparse vegetation growth achieves its peak performance, provide its greatest ability of cooling below the SUHI surface.

Pixels within the other two land cover categories observed a much larger ratio of negative residuals throughout the growing season, especially in summer and autumn. Forests and wetlands, which usually have low LST due to its dense vegetation compared to crop lands which contain more sparse vegetation along with bare soil, are known to behave as cool areas within a 
UHI (Sun, Wu, and Tan 2012). In the spring, forested land covers deviated the least of any season $\left(-0.27^{\circ} \mathrm{C}\right.$ compared to $0.54^{\circ} \mathrm{C}$ in the summer) from the UHI temperature mean in Wuhan, China from 2005 to 2015 (Q. Huang et al. 2019). The densely vegetated land covers surrounding Milton behave in a similar manner with its most effective cooling abilities occurring after spring.

4.2.2 Residuals Buffer Analysis: Figure 7 shows the density of mean SUHI residual values for each land cover within the buffer regions. The buffer analysis indicates the spatial distribution of residuals from each SUHI image as distance increases from the urban centre perimeter. Applying the results from the UHIFP (rural area 1.4 times the size of the urban area), the first three buffers are within the local footprint of the UHI.
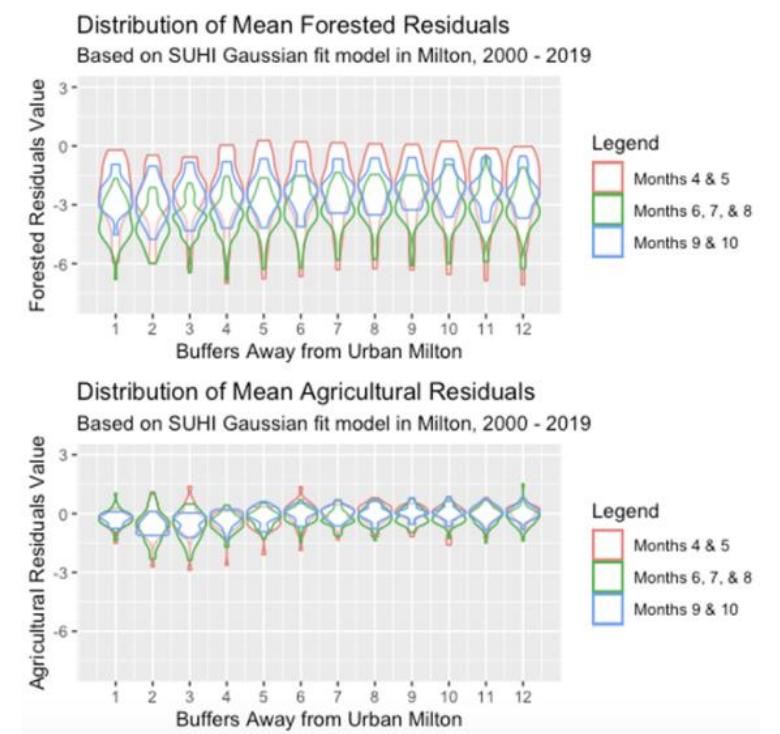

019

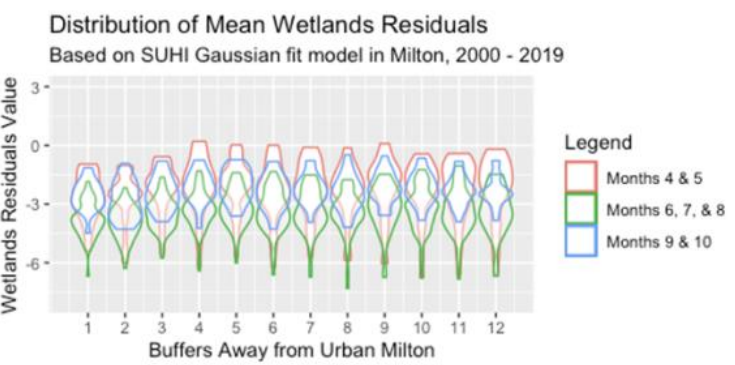

Distribution of Mean Urban Residuals

Based on SUHI Gaussian fit model in Milton, 2000 - 2019

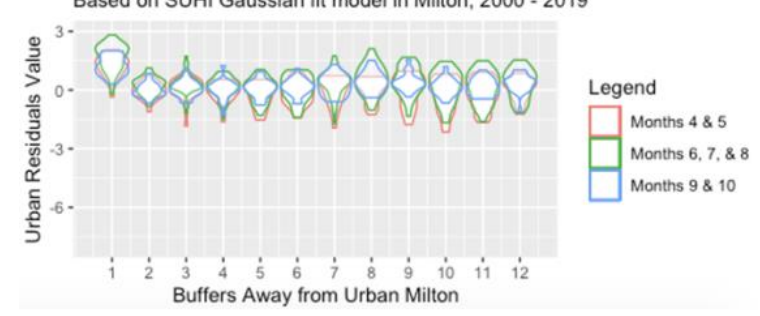

Figure 7. Density plot of the results from the buffers analysis with the residuals of each land cover within the SUHI models.

Seasonality plays a major role in determining the cooling and warming abilities within a UHI with dense vegetation and urban lands. Summer is when impervious surfaces are warmest and deviate most from the Gaussian surface. Forests and wetlands, in contrast, provide their greatest cooling effect during summer and deviate most while moderately reducing its cooling abilities in autumn. Agriculture exhibits minimal seasonal effects within Milton's UHI.

The buffer analysis with both forested and wetlands pixels determined a cooling effect which is much more evident within the first three buffers and in the main summer months. Throughout the year, the spatial influence which densely vegetated surfaces have on the UHI in Milton are most intense within the surrounding area 1.5 times the size of the urban centre (first 3 buffers), the rural area within the UHIFP. They remain definitive urban cool zones throughout the dataset with the highest mean residual value $(-2.02 \mathrm{~K})$ existing within the wetlands category at the fourth buffer zone during Spring. While green spaces within urban environments are known for the ability to improve local air quality, assist in the reduction of energy required for cooling, and optimize the natural ecological system (Ozyavuz 2012), the dense vegetation is most intense within the UHIFP.
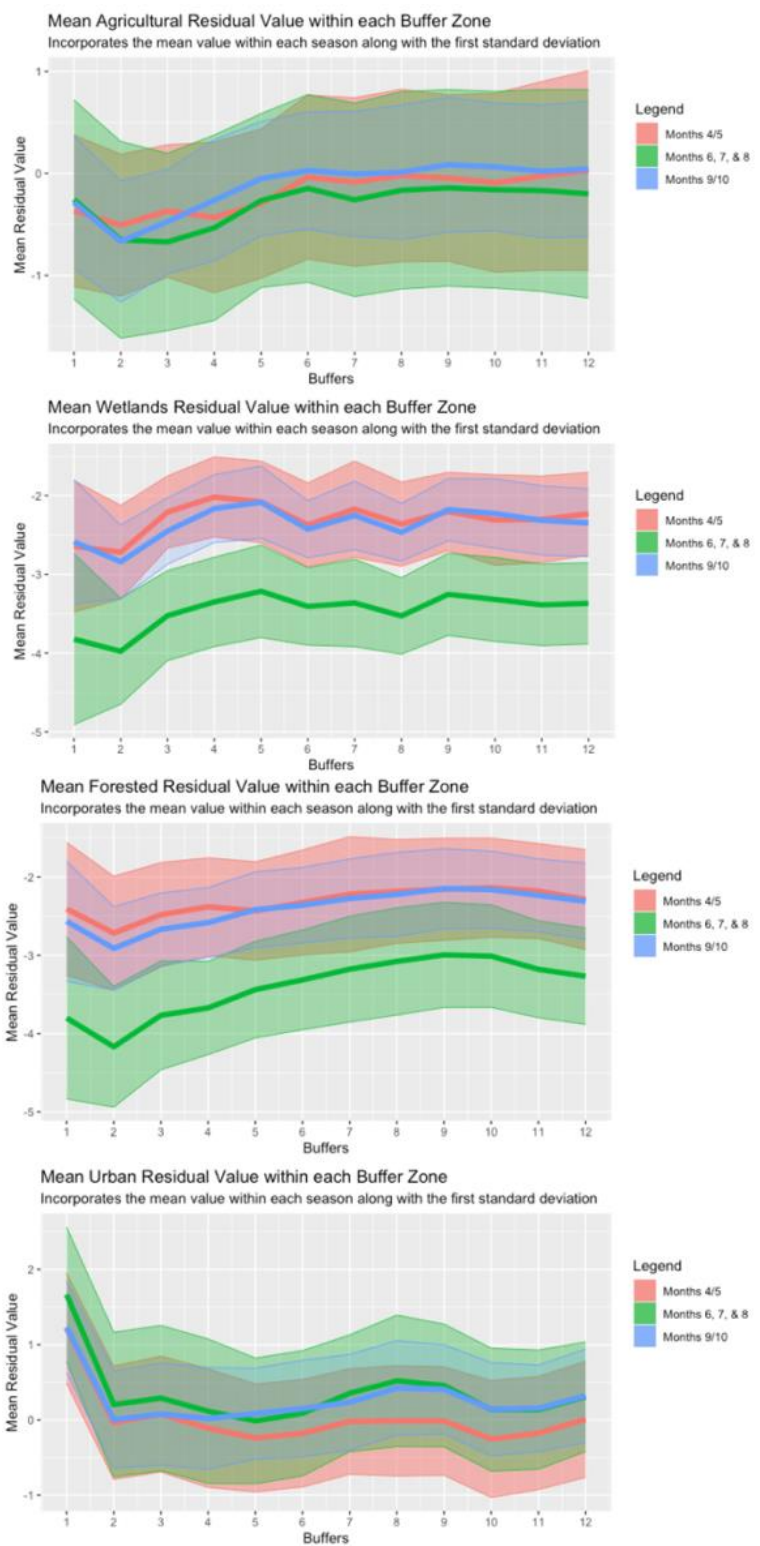

Figure 8. The mean and first standard deviation residual value of each land cover within each season for each buffer away from the central urban perimeter. 
Agricultural land covers convey similar observations to the more densely vegetated pixels. Their respective cooling effects are limited to the first five buffers within every season and maximized within the first three (the area within the UHIFP). The mean residual values ranged between $-0.67 \mathrm{~K}$ and $-0.26 \mathrm{~K}$ within the first 5 (4 for autumn) buffers away from the urban centre before stabilizing with the Gaussian surface. Being the dominant land cover of the region, the distribution is more likely to contribute to the SUHI planar fit and produce residuals closest to zero, especially during the time before planting and after cultivation where its consistency is largely bare soil. The summer months contributed slightly more cooling as a result of its peak vegetated state (Figure 8), especially within the outer buffers.

The reaction within the first buffer zone for the urban land covers' residuals is due to this paper's methods of obtaining an urban perimeter. The urban centre was calculated excluding transportation within UM census tracts and joined with a $15 \mathrm{~m}$ buffer to replace the missing roadways and obtain a centralized urban centre. As a result, heavy traffic located on the major 401 highway along with the immediate outer roads are the main contributors to the first buffer's urban distribution.

Urban results provide the highest LST and, aside from Spring, reliably positive residuals. For the remaining buffers, the average urban residuals conform closely to the Gaussian planar fit until the final 6 buffers away from the urban centre where its positivity increases (especially during the summer and autumn months). The area located outside of Milton's UHIFP (buffers 4 -12) is representative of where the ecological footprint that Milton has on its surrounding vegetation phenology has decayed (X. Zhang et al. 2004). The positive residual values that urban pixels experience outside of the UHIFP indicates a localized warming divergence from the Gaussian surface, which has decayed.

4.2.3 Time Frames Analysis: Mean LST for each time frame as each of the land cover datasets were used to determine general trends. Table 2 presents the SUHI magnitude, model thresholds, and the total quantity of available images within the region for each section of the growing season.

Results with SUHI magnitudes, thresholds, and measures of fit experience growth between the first and last time frame. An apparent rising SUHI magnitude is displayed within the first three time frames during the spring $(3.93 \mathrm{~K}$ to $6.67 \mathrm{~K})$ and summer $(6.55 \mathrm{~K}$ to $8.09 \mathrm{~K})$ of Table 2 before exhibiting a slight UHI magnitude reduction in the final time frame. The areas within each SUHI threshold experiences growth, especially in the final three time frames of each season.

Significant portions of the results do not indicate rising trends. The autumn months, with their limited quantities of clear sky pixels, provides uncharacteristically the largest thresholds within its first time frame. Fluctuations are also evident in the autumn's magnitudes throughout the research period.

The acquisition of mean LST data within each time frame presents complications with the results. A limited quantity of observations are available within each section of the growing season (16 to 26 available images for the summer months compared to 8 to 12 for the autumn months). The insufficiencies with the data results in bias and influence from outliers. With those considerations, even the period with the greatest potential quantity of observations (summer 2014-2019 has 26 observations) remains insufficient for decisive conclusions.

\begin{tabular}{|c|c|c|c|c|}
\hline & $\frac{2000-}{2003}$ & $\frac{2004-}{2008}$ & $\underline{2009-}$ & $\underline{2014-}$ \\
\hline $\begin{array}{l}\text { Months 4/5 } \\
\text { Magnitude (k) }\end{array}$ & 3.93 & 4.78 & 6.67 & 5.14 \\
\hline Months 4/5 & $15,028 /$ & $15,876 /$ & 21,656 & $32,789 /$ \\
\hline $\begin{array}{l}\text { Thresholds (Pixels) } \\
{\left[\mathrm{e}^{-1 / 2} / 1.0 \mathrm{~K}\right]}\end{array}$ & 41,151 & 49,658 & $\begin{array}{c}/ \\
82,202\end{array}$ & 107,341 \\
\hline $\begin{array}{l}\text { Months 4/5 } \\
\text { Available Images }\end{array}$ & 11 & 11 & 11 & 15 \\
\hline $\begin{array}{l}\text { Months 6/7/8 } \\
\text { Magnitude (K) }\end{array}$ & 6.55 & 6.92 & 8.09 & 7.81 \\
\hline Months 6/7/8 & $17,600 /$ & $17,043 /$ & 17,761 & $28,505 /$ \\
\hline $\begin{array}{l}\text { Thresholds (Pixels) } \\
{\left[\mathrm{e}^{-1 / 2} / 1.0 \mathrm{~K}\right]}\end{array}$ & 66,158 & 65,902 & $\begin{array}{c}/ \\
78,457\end{array}$ & 117,164 \\
\hline $\begin{array}{l}\text { Months 6/7/8 } \\
\text { Available Images }\end{array}$ & 16 & 22 & 22 & 26 \\
\hline $\begin{array}{l}\text { Months 9/10 } \\
\text { Magnitude (K) }\end{array}$ & 3.48 & 5.11 & 4.09 & 5.74 \\
\hline Months 9/10 & $48,299 /$ & $14,393 /$ & $25,610 /$ & $29,394 /$ \\
\hline $\begin{array}{l}\text { Thresholds (Pixels) } \\
{\left[\mathrm{e}^{-1 / 2} / 1.0 \mathrm{~K}\right]}\end{array}$ & 120,381 & 46,960 & 72,078 & 102,721 \\
\hline $\begin{array}{l}\text { Months } 9 / 10 \\
\text { Available Images }\end{array}$ & 8 & 9 & 10 & 12 \\
\hline
\end{tabular}

Table 2. The results from the time frames analysis which incorporated acquiring averaged imagery from each time period. It should be noted that the quantity of possible observations is attributed to the number of products available within each tile, thus not every observation is incorporated into the area of the study area.

4.2.4 Vegetation and Moisture Index Correlations: The results from the correlation analysis between the SUHI model's residuals and the moisture index (Figure 9) provide the most consistently positive correlation results with all land cover categories. Wetlands and agriculture provided the strongest relationships throughout the growing season along with significantly positive results with urban pixels in the summer. In spring months, the strongest corrections occurs in the wetlands (0.52) while the weakest ones appears in urban land (0.31). Summer has agriculture being the strongest $(0.74)$ with urban surfaces being second strongest (0.56). In autumn, agriculture and wetlands have stronger relationships $(0.62$ and 0.58 respectfully) and urban surfaces were the weakest again with moderate results (0.45). 


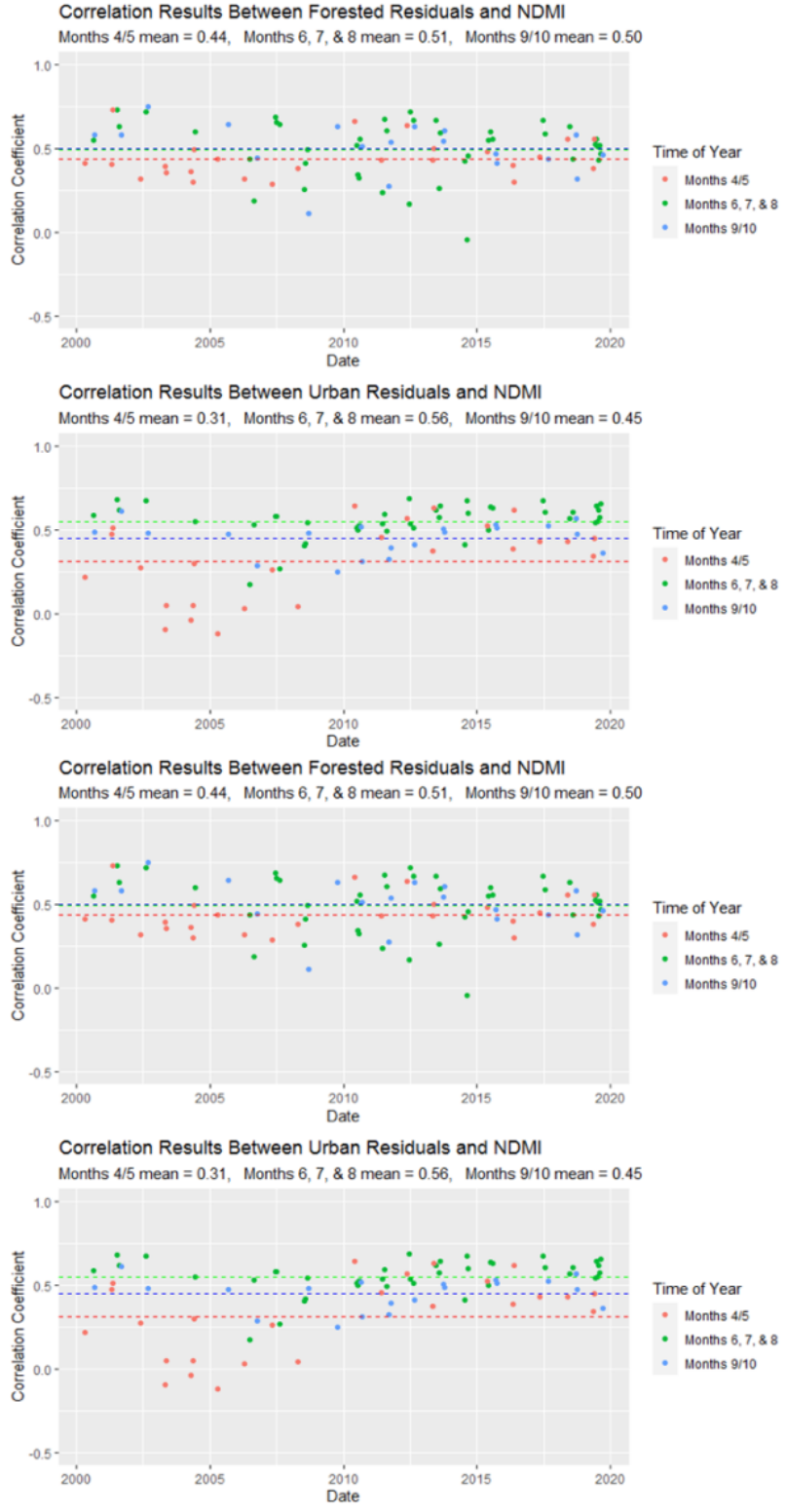

Figure 9. The correlation coefficient each land cover's residuals have with the NDMI product with the same Landsat 7 product. This comparison was determined to have the strongest correlation with the residuals of every land cover.

The results with EVI (Table 3) indicate stronger relationships with dense vegetation in the early growing season. Average wetlands and forested results in the spring are 0.44 and 0.42 respectfully compared to 0.36 in agriculture. The mean correlation coefficients for the remainder of the growing season are similar with all three vegetated surfaces (between 0.38 and 0.41 in the summer and 0.27 to 0.30 in the autumn). Urban pixels experienced their weakest correlations with this vegetation index.

The results with NDVI (Table 3) describe a strong relationship with agriculture while moderately weaker with dense vegetation and low-density urban surfaces in the summer and autumn months. Sparse vegetation experienced similar correlations to NDVI as dense vegetation in the spring months (mean values between 0.36 and 0.39 ) before providing significantly stronger results throughout the remainder of the year $(0.67$ for the summer and 0.53 in autumn). Urban pixels, alternatively, had weak relationships in the early growing season before providing similar results to dense vegetation in the summer and autumn.

\begin{tabular}{|l|c|c|c|c|}
\hline & Agricultural & $\underline{\text { Forested }}$ & $\underline{\text { Wetlands }}$ & $\underline{\text { Urban }}$ \\
\hline $\begin{array}{l}\text { EVI } \\
\text { (M. 4/5) }\end{array}$ & 0.36 & 0.42 & 0.44 & 0.19 \\
\hline $\begin{array}{l}\text { EVI } \\
\text { (M. 6-8) }\end{array}$ & 0.40 & 0.38 & 0.41 & 0.28 \\
\hline $\begin{array}{l}\text { EVI } \\
\text { (M. 9/10) }\end{array}$ & 0.27 & 0.27 & 0.30 & 0.18 \\
\hline $\begin{array}{l}\text { NDVI } \\
\text { (M. 4/5) }\end{array}$ & 0.39 & 0.36 & 0.37 & 0.29 \\
\hline $\begin{array}{l}\text { NDVI } \\
\text { (M. 6-8) }\end{array}$ & 0.67 & 0.42 & 0.46 & 0.49 \\
\hline $\begin{array}{l}\text { NDVI } \\
\text { (M. 9/10) }\end{array}$ & 0.53 & 0.39 & 0.47 & 0.40 \\
\hline $\begin{array}{l}\text { NDMI } \\
\text { (M. 4/5) }\end{array}$ & 0.47 & 0.44 & 0.52 & 0.31 \\
\hline $\begin{array}{l}\text { NDMI } \\
\text { (M. 6-8) }\end{array}$ & 0.74 & 0.51 & 0.54 & 0.56 \\
\hline $\begin{array}{l}\text { NDMI } \\
\text { (M. 9/10) }\end{array}$ & 0.62 & 0.50 & 0.58 & 0.45 \\
\hline
\end{tabular}

Table 3. The results from a correlation analysis with each of the indices. The summary statistics are similar to Figure 9 with only the inclusion of mean values from each set of months (M.).

Moisture was consistently the strongest variable affecting the SUHI residuals over every land cover. NDMI is considered as a cooling index useful for regulating thermal configuration with strong greening properties (Zhu et al. 2019). With a consistent moisture distribution throughout the study area, NDMI is capable of retaining a strong correlation with every bioproductive land cover throughout every season. Amongst all indices, its correlation with urban pixels was the strongest due to Milton's low-density residential distribution. Built-up pervious land in the form of lawns, parks, and recreational areas increase abilities for moisture retention.

The land cover which obtained the weakest correlation results with both vegetation indices was urban. This is due to the distribution of impervious surfaces, contrasting vegetation land covers and their abilities to retain moisture and reflect more infrared radiation, which are important in all three indices. The inclusion of built-up pervious land covers (in reference to urban recreation areas, such as golf courses or playing fields (Science and Research Branch of the Ministry of Natural Resources and Forestry 2019)) may be the primary contributor to the positive correlations. Additionally, the low-density structure of the residential neighbourhoods allow for a reduced distribution of impervious surfaces and increased abilities for infrared reflection, sparse vegetation distribution, and moisture retention. As a result, as noted in Table 3, urban surfaces correlate relatively well with NDVI in summer and autumn.

Agricultural surfaces dominated the correlations with NDVI and NDMI with mean correlation coefficients as high as 0.64 and 0.74 within the summer months respectfully. With EVI, the forested and wetlands surfaces obtained a marginally higher correlation, with April and May results deviating the most in Table 3. Considering that LST generally trends in contrast to NDVI and EVI (Alademomi et al. 2020), the stronger correlation with EVI is due to the denser vegetation and reduced soil reflectance while agriculture is distributed more dominantly and performs better with NDVI. 


\section{CONCLUSION}

The two common methods for analysing the footprint of a UHI was adapted for a rapidly urbanizing low-density urban area in Canada using LST acquired from Landsat 7 ETM+. Both UHIFP and SUHI models were used for a comparison analysis and to determine the primary factors influencing the results. These techniques allowed for an enhanced comprehension of the effects that the local UHI has upon rural (or exurban) vegetation within differing bio-productive land covers with varying degrees of distance applied.

Milton, a secondary (or suburban) city, was determined to have an average rural UHIFP 1.4 times the size of the urban centre. It is smaller than previous research due to the study site's dwarfed size compared to previous studies, reduced population density, its proximity to larger urban centres at its periphery which influence the rural reference LST, and the rural vegetation distribution. Additionally, the use of 5 years mean imagery for Landsat 7 ETM+ provided an effective and consistent method for calculating the UHIFP with a 16-days temporal resolution.

Despite agricultural surfaces being the most dominantly distributed throughout the region, urban land covers dominated influencing the UHIFP and SUHI models. It mainly followed the SUHI Gaussian surface and exhibited increased warming effects outside of the UHIFP. Agricultural surfaces achieved their greatest cooling effects in the area 2.5 times the urban centre area. Alternatively, densely vegetated land covers maximized their cooling abilities within the rural area 1.5 times the size of the urban centre.

NDMI provided the strongest correlations with every land cover throughout every part of the growing season. As a result, moisture may be more important to the spatial pattern of the $\mathrm{UHI}$ and its footprint than vegetation health.

The results from this analysis describe the impact which small scale urban expansion poses for surrounding rural environments. The plethora of proximate agricultural surfaces and distribution of dense vegetation presents Milton's surroundings as an ideal representation for suburban communities across Canada and abroad. Conclusions made here are very useful for city planners, engineers, and geographers in their abilities to predict the impact of low-density urban expansion and its spatiotemporal influence. The strong correlations urban residuals found with NDMI and NDVI defines how the increased vegetation distributions in lowdensity neighbourhoods and urban centres influences the local $\mathrm{UHI}$ and rural vegetation.

\section{ACKNOWLEDGEMENTS}

The authors are grateful for Dr. Stefania Bonafoni and Roberta Anniballe's contribution of the MATLAB script for determining the SUHI Gaussian planar fit model based on their previous research.

This research was supported by a National Science and Engineering Research Council (NSERC) Discovery Grant and Queen's Graduate Scholarship.

\section{REFERENCES}

Adamowski, Jan, and Andreas Prokoph. 2013. "Assessing the Impacts of the Urban Heat Island Effect on Streamflow Patterns in Ottawa, Canada.” Journal of Hydrology 496 (July): 225-37. https://doi.org/10.1016/j.jhydrol.2013.05.032.

Alademomi, Alfred S., Chukwuma J. Okolie, Olagoke E. Daramola, Raphael O. Agboola, and Tosin J. Salami. 2020. "Assessing the Relationship of LST, NDVI and EVI with Land Cover Changes in the Lagos Lagoon Environment."

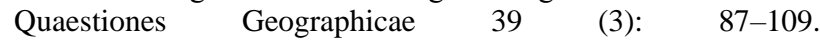
https://doi.org/10.2478/quageo-2020-0025.

Anniballe, Roberta, and Stefania Bonafoni. 2015. "A Stable Gaussian Fitting Procedure for the Parameterization of Remote Sensed Thermal Images." Algorithms 8 (2): 82-91. https://doi.org/10.3390/a8020082.

Anniballe, Roberta, Stefania Bonafoni, and Manuele Pichierri. 2014. "Spatial and Temporal Trends of the Surface and Air Heat Island over Milan Using MODIS Data." Remote Sensing

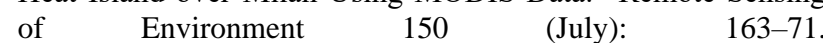
https://doi.org/10.1016/j.rse.2014.05.005.

Bernhardt, Jase, and Andrew M. Carleton. 2019. "Comparing Daily Temperature Averaging Methods: The Role of Surface and Atmosphere Variables in Determining Spatial and Seasonal Variability." Theoretical \& Applied Climatology 136 (1/2): 499-512. https://doi.org/10.1007/s00704-018-2504-7.

Ermida, Sofia L., Patricia Soares, Vasco Mantas, Frank-M. Gottsche, and Isabel F. Trigo. 2020. "Google Earth Engine Open-Source Code for Land Surface Temperature Estimation from the Landsat Series.” Journal of Remote Sensing 12 (9). https://doi.org/10.3390/rs12091471.

Gordon, David L. A., and Mark Janzen. 2013. "Suburban Nation? Estimating the Size of Canada's Suburban Population." Journal of Architectural and Planning Research 30 (3).

Guillevic, Pierre, Frank Gottsche, Jaime Nickeson, Glynn Hulley, Darren Ghent, Yunyue Yu, Isabel Trigo, et al. 2018. "Land Surface Temperature Product Validation Best Practice Protocol." Version 1.1 https://lpvs.gsfc.nasa.gov/PDF/CEOS_LST_PROTOCOL_Feb2 018_v1.1.0_light.pdf.

Hennessey, Melanie. 2017. "Census: Milton Remains in Top 10 Fastest-Growing Canadian Communities." Inside Halton, February 8, 2017, sec. Milton Canadian Champion. https://www.insidehalton.com/news-story/7110264-censusmilton-remains-in-top-10-fastest-growing-canadiancommunities/.

Howard, Luke. 1818. The Climate of London: Deduced from Meteorological Observations, Made at Different Places in the Neighbourhood of the Metropolis. W. Phillips, George Yard, Lombard Street, sold also by J. and A. Arch, Cornhill; Baldwin, Cradock, and Joy, and W. Bent, Paternoster Row; and J. Hatchard, Picadilly.

Huang, Huanchun, Yingxia Yun, Hao Xu, and Ting Liu. 2019. "Influence of the Mega-Urban Heat Island on Spatial Transfer of Summer Thermal Comfort: Evidence from Tianjin, China." Tehnicki Vjesnik - Technical Gazette 26 (1): 183-93. https://doi.org/10.17559/TV-20181108143831.

Huang, Qiuping, Jiejun Huang, Xining Yang, Chuanglin Fang, and Youjia Liang. 2019. "Quantifying the Seasonal Contribution of Coupling Urban Land Use Types on Urban Heat Island Using Land Contribution Index: A Case Study in 
Wuhan, China." Sustainable Cities and Society 44 (January): 666-75. https://doi.org/10.1016/j.scs.2018.10.016.

Imhoff, Marc L., Ping Zhang, Robert E. Wolfe, and Lahouari Bounoua. 2010. "Remote Sensing of the Urban Heat Island Effect across Biomes in the Continental USA." Remote Sensing $\begin{array}{llll}\text { of } & \text { Environment } & 114 & \text { (3): }\end{array}$ https://doi.org/10.1016/j.rse.2009.10.008.

Katsoulis, B. D., and G. A. Theoharatos. 1985. "Indications of the Urban Heat Island in Athens, Greece." Journal of Climate and Applied Meteorology 24 (12): 1296-1302.

Krehbiel, Cole P., Trisha Jackson, and Geoffrey M. Henebry. 2016. "Web-Enabled Landsat Data Time Series for Monitoring Urban Heat Island Impacts on Land Surface Phenology." IEEE Journal of Selected Topics in Applied Earth Observations and Remote Sensing 9 (5): 2043-50. https://doi.org/10.1109/JSTARS.2015.2496951.

Lee, Harold, Wasyf Bakowsky, John Riley, Jane Bowles, Michael Puddister, Peter Uhlig, and Sean McMurray. 1998. "Ecological Land Classification for Southern Ontario." SCSS Field Guide FG-02. North Bay: Ontario Ministry of Natural Resources.

Li, Ying-ying, Hao Zhang, and Wolfgang Kainz. 2012. "Monitoring Patterns of Urban Heat Islands of the FastGrowing Shanghai Metropolis, China: Using Time-Series of Landsat TM/ETM+ Data." International Journal of Applied Earth Observation and Geoinformation 19 (October): 127-38. https://doi.org/10.1016/j.jag.2012.05.001.

Lu, Dongmei, Kaishan Song, Shuying Zang, Mingming Jia, Jia Du, and Chunying Ren. 2015. "The Effect of Urban Expansion on Urban Surface Temperature in Shenyang, China: An Analysis with Landsat Imagery." Environmental Modeling \& Assessment 20 (3): 197-210. https://doi.org/10.1007/s10666014-9426-2.

Malakar, Nabin K., Glynn C. Hulley, Simon J. Hook, Kelly Laraby, Monica Cook, and John R. Schott. 2018. "An Operational Land Surface Temperature Product for Landsat Thermal Data: Methodology and Validation." IEEE Transactions on Geoscience and Remote Sensing 56 (10): 5717-35. https://doi.org/10.1109/TGRS.2018.2824828.

Maoh, Hanna, and Pavlos Kanaroglou. 2007. "Geographic Clustering of Firms and Urban Form: A Multivariate Analysis." Journal of Geographical Systems 9 (1): 29-52. http://dx.doi.org.proxy.queensu.ca/10.1007/s10109-006-0029-6. Martin, Philippe, Yves Baudouin, and Philippe Gachon. 2015. "An Alternative Method to Characterize the Surface Urban Heat Island." International Journal of Biometeorology 59 (7): 849 61. https://doi.org/10.1007/s00484-014-0902-9.

Meng, Chunlei, and Youjun Dou. 2016. "Quantifying the Anthropogenic Footprint in Eastern China." Scientific Reports 6 (1): 24337. https://doi.org/10.1038/srep24337.

Meng, F., and M. Liu. 2013. "Remote-Sensing Image-Based Analysis of the Patterns of Urban Heat Islands in Rapidly Urbanizing Jinan, China." International Journal of Remote $\begin{array}{llll}\text { Sensing } & 34 & \text { (24): } & \text { 8838-53. }\end{array}$ https://doi.org/10.1080/01431161.2013.853895.
Ministry of Agriculture, Food and Rural Affairs. 2020. "Climate Zones and Planting Dates for Vegetables in Ontario." Government of Ontario. August 7, 2020. http://www.omafra.gov.on.ca/english/crops/facts/climzoneveg.h tm.

National Aeronautics and Space Administration. 2020. "Landsat 7 Science Data Users Handbook." NASA. https://landsat.gsfc.nasa.gov/wp-

content/uploads/2016/08/Landsat7_Handbook.pdf.

Oke, T. R. 1973. "City Size and the Urban Heat Island." Atmospheric Environment (1967) 7 (8): 769-79. https://doi.org/10.1016/0004-6981(73)90140-6.

1979. "Advectively-Assisted Evapotranspiration from Irrigated Urban Vegetation.” Boundary-Layer Meteorology 17 (2): 167-73. https://doi.org/10.1007/BF00117976.

Ozyavuz, Murat. 2012. Landscape Planning. IntechOpen, pp. 107-122.

Qiao, Zhi, Chen Wu, Dongqi Zhao, Xinliang Xu, Jilin Yang, Li Feng, Zongyao Sun, and Luo Liu. 2019. "Determining the Boundary and Probability of Surface Urban Heat Island Footprint Based on a Logistic Model.” Remote Sensing 11 (11): 1368. https://doi.org/10.3390/rs11111368.

Ramírez-Aguilar, Edwin Alejandro, and Léa Cristina Lucas Souza. 2019. "Urban Form and Population Density: Influences on Urban Heat Island Intensities in Bogotá, Colombia." Urban $\begin{array}{llll}\text { Climate } & 29 & \text { (September): } & 100497 .\end{array}$ https://doi.org/10.1016/j.uclim.2019.100497.

Sampson, Paul. 2007. "SOLRIS Accuracy Assessment Report 1: Golden Horseshoe Area (SOLRIS Study Area A)." Accuracy Assessment 1 (Version 2.0). Peterborough, ON: Ministry of Natural Resources.

Science and Research Branch of the Ministry of Natural Resources and Forestry. 2019. "Southern Ontario Land Resource Information System (SOLRIS) Version 3.0: Data Specifications." Ministry of Natural Resources.

Skelhorn, Cynthia P., Geoff Levermore, and Sarah J. Lindley. 2016. "Impacts on Cooling Energy Consumption Due to the UHI and Vegetation Changes in Manchester, UK." Energy and Buildings 122 (June): 150-59. https://doi.org/10.1016/j.enbuild.2016.01.035.

Sobrino, J. A., R. Oltra-Carrió, G. Sòria, R. Bianchi, and M. Paganini. 2012. "Impact of Spatial Resolution and Satellite Overpass Time on Evaluation of the Surface Urban Heat Island Effects." Remote Sensing of Environment, Remote Sensing of Urban Environments, 117 (February): 50-56. https://doi.org/10.1016/j.rse.2011.04.042.

Streutker, D. R. 2002. "A Remote Sensing Study of the Urban Heat Island of Houston, Texas." International Journal of Remote Sensing $23 \quad$ (13): 2595-2608. https://doi.org/10.1080/01431160110115023.

Streutker, David R. 2003. "Satellite-Measured Growth of the Urban Heat Island of Houston, Texas." Remote Sensing of Environment 85 (3): 282-89. https://doi.org/10.1016/S00344257(03)00007-5. 
Sun, Qinqin, Zhifeng Wu, and Jianjun Tan. 2012. "The Relationship between Land Surface Temperature and Land Use/Land Cover in Guangzhou, China." Environmental Earth Sciences 65 (6): 1687-94. https://doi.org/10.1007/s12665-011$1145-2$.

Tomlinson, Charlie J., Lee Chapman, John E. Thornes, and Christopher Baker. 2011. "Remote Sensing Land Surface Temperature for Meteorology and Climatology: A Review." Meteorological Applications 18 (3): 296-306. https://doi.org/10.1002/met.287.

Touchaei, A. G., and Y. Wang. 2015. "Characterizing Urban Heat Island in Montreal (Canada)_Effect of Urban Morphology." Sustainable Cities and Society 19 (December): 395-402. https://doi.org/10.1016/j.scs.2015.03.005.

Wang, Kaicun, Shaojing Jiang, Jiankai Wang, Chunlüe Zhou, Xiaoyan Wang, and Xuhui Lee. 2017. "Comparing the Diurnal and Seasonal Variabilities of Atmospheric and Surface Urban Heat Islands Based on the Beijing Urban Meteorological Network." Journal of Geophysical Research: Atmospheres 122 (4): 2131-54. https://doi.org/10.1002/2016JD025304.

Wang, Yupeng, Umberto Berardi, and Hashem Akbari. 2016. "Comparing the Effects of Urban Heat Island Mitigation Strategies for Toronto, Canada." Energy and Buildings, SI: Countermeasures to Urban Heat Island, 114 (February): 2-19. https://doi.org/10.1016/j.enbuild.2015.06.046.

Yang, Qiquan, Xin Huang, and Qiuhong Tang. 2019. "The Footprint of Urban Heat Island Effect in 302 Chinese Cities: Temporal Trends and Associated Factors." Science of The Total Environment 655 (March): 652-62. https://doi.org/10.1016/j.scitotenv.2018.11.171.

Yao, Rui, Lunche Wang, Xuan Gui, Yukun Zheng, Haoming Zhang, and Xin Huang. 2017. "Urbanization Effects on Vegetation and Surface Urban Heat Islands in China's Yangtze River Basin." Remote Sensing 9 (6): 540. https://doi.org/10.3390/rs9060540.

Yao, Rui, Lunche Wang, Xin Huang, Wei Gong, and Xiangao Xia. 2019. "Greening in Rural Areas Increases the Surface Urban Heat Island Intensity." Geophysical Research Letters 46 (4): 2204-12. https://doi.org/10.1029/2018GL081816.

Yu, Zhaowu, Yawen Yao, Gaoyuan Yang, Xiangrong Wang, and Henrik Vejre. 2019. "Strong Contribution of Rapid Urbanization and Urban Agglomeration Development to Regional Thermal Environment Dynamics and Evolution." Forest Ecology and Management 446 (August): 214-25. https://doi.org/10.1016/j.foreco.2019.05.046.

Zhang, Da-Lin, Yi-Xuan Shou, Russell R. Dickerson, and Fei Chen. 2011. "Impact of Upstream Urbanization on the Urban Heat Island Effects along the Washington-Baltimore Corridor." Journal of Applied Meteorology and Climatology 50 (10): 2012-29.

Zhang, Xiaoyang, Mark A. Friedl, Crystal B. Schaaf, Alan H. Strahler, and Annemarie Schneider. 2004. "The Footprint of Urban Climates on Vegetation Phenology." Geophysical $\begin{array}{llll}\text { Research } & \text { Letters } & & \\ & \end{array}$ https://doi.org/10.1029/2004GL020137.
Zhou, Decheng, Jingfeng Xiao, Stefania Bonafoni, Christian Berger, Kaveh Deilami, Yuyu Zhou, Steve Frolking, Rui Yao, Zhi Qiao, and José A. Sobrino. 2019. "Satellite Remote Sensing of Surface Urban Heat Islands: Progress, Challenges, and Perspectives." Remote Sensing $11 \quad$ (1): 48. https://doi.org/10.3390/rs11010048.

Zhou, Decheng, Shuqing Zhao, Liangxia Zhang, Ge Sun, and Yongqiang Liu. 2015. "The Footprint of Urban Heat Island Effect in China." Scientific Reports 5 (1): 1-11. https://doi.org/10.1038/srep11160.

Zhou, Weiqi, Yuguo Qian, Xiaoma Li, Weifeng Li, and Lijian Han. 2014. "Relationships between Land Cover and the Surface Urban Heat Island: Seasonal Variability and Effects of Spatial and Thematic Resolution of Land Cover Data on Predicting Land Surface Temperatures." Landscape Ecology 29 (1): 15367. https://doi.org/10.1007/s10980-013-9950-5.

Zhu, Xinming, Xuhong Wang, Dajiang Yan, Zhuang Liu, and Yongfang Zhou. 2019. "Analysis of Remotely-Sensed Ecological Indexes' Influence on Urban Thermal Environment Dynamic Using an Integrated Ecological Index: A Case Study of Xi'an, China." International Journal of Remote Sensing 40 (9): 3421-47. https://doi.org/10.1080/01431161.2018.1547448. 